\title{
Traumatismo Cranioencefálico Grave em Crianças e Adolescentes*
}

\section{Severe Traumatic Brain Injury in Children and Adolescents}

\author{
Luís Fernando Andrade de Carvalho ${ }^{1}$, Carolina de Araújo Affonseca², \\ Sérgio Diniz Guerra ${ }^{3}$, Alexandre Rodrigues Ferreira ${ }^{4}$, Eugênio Marcos Andrade Goulart4.
}

\section{RESUMO}

JUSTIFICATIVA E OBJETIVOS: Apresentar uma revisão crítica do traumatismo cranioencefálico (TCE) em crianças e adolescentes, enfocando o trauma grave, as taxas e os aspectos relacionados com a mortalidade e as principais medidas terapêuticas.

CONTEÚDO: Revisão sistemática dos artigos incluídos no MEDLINE, SciELO e Lilacs, no período compreendido entre 1985 e 2006, utilizando as seguintes palavraschave: traumatic brain injury, craniocerebral trauma, children e mortality, além dos principais livros-texto sobre 0 assunto. Foram analisados também os Guidelines of Brain Trauma Foundation de 2000 e 2003, sendo selecionados os principais trabalhos citados. $\mathrm{O}$ traumatismo cranioencefálico é uma das principais causas de morte e seqüelas em crianças e adolescentes. As taxas de mortalidade variaram entre $10 \%$ e $55 \%$, principalmente em razão dos critérios de seleção utilizados, da gravidade dos pacientes e dos locais onde foram realizados os estudos. Em geral, a mortalidade em crianças foi menor que as descritas em pacientes adultos. Os principais fa-

1. Mestre em Pediatria, Intensivista Pediátrico, Diarista da UTI Pediátrica do Hospital João XXIII / FHEMIG.

2. Mestre em Pediatria, Intensivista Pediátrica, Médica do SAMU / Belo Horizonte.

3. Mestre em Pediatria, Intensivista Pediátrico, Coordenador da UTI do Hospital João XXIII / FHEMIG.

4. Doutor em Pediatria, Professor Adjunto da UFMG.

*Recebido do Hospital João XXIII da FHEMIG, Belo Horizonte, MG

Apresentado em 18 de agosto de 2006.

Aceito para publicação em 22 de fevereiro de 2007

Endereço para correspondência:

Dr. Luís Fernando Andrade de Carvalho

Rua Heitor Sócrates Cardoso, 630 - Santa Amélia

31560-320 Belo Horizonte, MG

Fone: (31) 3427-5219 Fax: (31) 3427-5219

E-mail: luiscarol@uol.com.br

(CAssociação de Medicina Intensiva Brasileira, 2007 tores relacionados com a mortalidade em crianças foram a pontuação na escala de coma de Glasgow, a hipotensão arterial, o edema e ingurgitamento cerebral difusos e a baixa pressão de perfusão encefálica. $O$ tratamento do TCE grave visa a correção dos fatores relacionados com a lesão cerebral secundária.

CONCLUSÕES: Os fatores relacionados com melhor prognóstico em crianças ainda permanecem inconclusivos, havendo algumas divergências entre os estudos. Grande parte destes fatores é corrigível ou evitável, com reanimação hídrica inicial agressiva, tratamento cirúrgico em tempo hábil, monitorização e cuidados intensivos adequados.

Unitermos: adolescente, criança, mortalidade, tratamento, trauma craniocerebral

\section{SUMMARY}

BACKGROUND AND OBJECTIVES: Present a critical review of traumatic brain injury (TBI) in children and adolescents, focusing on severe TBI, the mortality and the factors related with poor outcome.

CONTENTS: It was made a systematic review in MEDLINE, SciElo e Lilacs, with the key words: traumatic brain injury, craniocerebral trauma, children and mortality. The most important articles related in the Guidelines of Brain Trauma Foundation (2000 e 2003) were selected too. TBI is one of the most important causes of mortality and morbidity in children and adolescents, and morbidity in children and adolescents. The mortality variation was between $10 \%$ and $55 \%$, depending of the patients select criteria, trauma severity and units where the studies were made. The children mortality was, in general, lower than that found in adults and the most important factors related with an increased mortality were: Glasgow Coma Score, hypotension, cerebral swelling and lower cerebral perfusion press. Severe TBI endpoint treatment is to correct the secondary brain lesions related factors. 
CONCLUSIONS: The factors related with better outcomes in children with severe TBI are still obscures, despite the large number of studies, large numbers of studies. Many of these factors can be avoided or correct by aggressive fluid resuscitation, surgery treatment, monitoration and adequate intensive care.

Key Words: adolescent, child, mortality, craniocerebral trauma, treatment

\section{INTRODUÇÃO}

O trauma é uma das principais causas de morte e de seqüelas em crianças e adolescentes no mundo'. No Brasil, é a principal causa de morte em crianças acima de cinco anos de idade e responsável por mais de $50 \%$ dos óbitos na adolescência ${ }^{2}$. O traumatismo cranioencefálico (TCE) está presente na maioria das crianças vítimas de trauma e é responsável por mais de $75 \%$ das mortes na infância ${ }^{1,3,4}$. Crianças com TCE grave necessitam de internação prolongada em Unidade de Terapia Intensiva (UTI), com alta mortalidade e morbidade, além de elevado custo financeiro ${ }^{5}$.

O conhecimento das causas do traumatismo cranioencefálico grave em crianças e adolescentes possibilita a implantação de medidas para prevenção primária, que visam à redução do número de traumas, ao passo que a determinação dos fatores relacionados com o óbito propicia a elaboração de protocolos de tratamento para reduzir a morbidade e a mortalidade, denominada prevenção secundária.

\section{CLASSIFICAÇÃO}

O TCE pode ser classificado quanto ao mecanismo, gravidade ou morfologia $a^{4,6-8}$.

- Mecanismo: a lesão pode ser fechada (contusa) ou penetrante. As lesões fechadas, mais comuns na infância, são causadas por quedas, atropelamentos, acidentes automobilísticos e agressões;

- Gravidade: é baseada na escala de coma de Glasgow (ECG), pode ser leve (ECG 14 e 15), moderada (ECG 9 a 13) ou grave (ECG 3 a 8) $)^{8}$. A ECG é utilizada também como parâmetro evolutivo e como índice prognóstico, devendo ser interpretada com cautela em pacientes com hipóxia, hipotensão arterial ou após o uso de sedativos e bloqueadores neuromusculares, álcool ou outros fármacos.

- Morfologia: divide-se em lesões extracranianas, fraturas do crânio e lesões intracranianas;

1) Lesões extracranianas: lacerações de couro cabelu- do que podem ser fonte importante de sangramento e hematomas subgaleais;

2) Fraturas de crânio: lineares, cominutivas, com afundamento, geralmente associadas com lesão de duramáter e do parênquima cerebral ou diastáticas. A ocorrência de fraturas aumenta significativamente o risco de lesões intracranianas ${ }^{4}$;

3) Lesões intracranianas: podem ser focais (hematomas extradural, subdural ou intra-parenquimatoso) ou difusas (concussão, lesão axonal difusa ou edema e ingurgitamento cerebral). As lesões difusas são as mais comuns em crianças com TCE, principalmente pela desproporção entre a cabeça e o tronco, que propicia a movimentação pendular, pela imaturidade encefálica com grande conteúdo de água e com mielinização incompleta e pela expressiva susceptibilidade às lesões secundárias ${ }^{9}$.

\section{LESÃO ENCEFÁLICA PRIMÁRIA E SECUNDÁRIA}

Lesão primária é o resultado direto da lesão mecânica provocada pelo trauma, podendo ser causada por dois mecanismos: pelo impacto ou por aceleração e desaceleração. Ocorre no momento do trauma e geralmente não pode ser amenizada pela intervenção médica e sim por meio de medidas de prevenção primária ${ }^{4}$. Já a lesão cerebral secundária é causada no SNC em decorrência da resposta fisiológica sistêmica ao trauma inicial. As causas mais comuns de lesão cerebral secundária são hipoxemia, hipercapnia ou hipocapnia, hipotensão arterial, hipertensão intracraniana, crises convulsivas, hipertermia e distúrbios hidroeletrolíticos e metabólicos, principalmente de sódio e glicose. A prevenção e a correção dos fatores causadores de lesão cerebral secundária são as medidas mais eficazes no tratamento da criança com TCE $4,6,7,9,10$.

\section{FATORES RELACIONADOS COM A MORTALIDADE}

A experiência clínica e os trabalhos científicos têm mostrado um prognóstico mais favorável nas crianças com TCE que nos adultos ${ }^{11-13}$. De forma geral, as taxas de mortalidade do TCE grave em Pediatria variam, entre os trabalhos publicados, de $10 \%$ a $50 \%{ }^{14-20}$. Essa ampla variação deve-se, em parte, à diferença dos métodos e dos critérios de seleção utilizados, bem como às diferenças inerentes a cada serviço de referência, ou seja: gravidade dos pacientes, atendimento préhospitalar, recursos disponíveis, entre outros.

Existe também variação nos fatores descritos como relacionados com a mortalidade de crianças com TCE 
grave. Os trabalhos publicados ${ }^{17-21}$ nas décadas de 1970 e 1980 validaram o uso da ECG em crianças, descrita por Teasdale e Jennett ${ }^{22}$ em 1974, bem como a sua relação com a mortalidade. Eles analisaram ainda alguns fatores, individualmente em análises univariadas, sugerindo uma possível relação com maior mortalidade, como: hipertensão intracraniana, hipotensão, hipóxia, hipercapnia, lesões intracranianas e traumatismos associados ao TCE, servindo como base para os estudos que surgiram nas duas últimas décadas.

Estudos mais recentes reafirmaram a importância da hipotensão no aumento da mortalidade ${ }^{5,14,23-26}$. A presença de hipertensão intracraniana, a hiperglicemia e os distúrbios de coagulação sanguínea, principalmente a coagulação intravascular disseminada estiveram relacionados, em alguns estudos ${ }^{14,18,25,27}$, com um pior prognóstico de crianças com TCE grave, assim como algumas lesões intracranianas, encontradas na tomografia de crânio, como o edema e o ingurgitamento cerebral difuso ${ }^{16,25}$, o hematoma subdural ${ }^{15}$, a hemorragia subaracnóidea ${ }^{28}$ e a lesão axonal difusa ${ }^{29}$.

A tabela 1 apresenta os principais estudos encontrados na literatura nos últimos 20 anos (1985-2006), que incluem as variáveis relacionadas com a mortalidade no traumatismo cranioencefálico grave em crianças e adolescentes.

Tabela 1 - Estudos sobre os Fatores Relacionados com Mortalidade de Crianças e Adolescentes com Trauma Cranioencefálico Grave (1985-2006).

\begin{tabular}{|c|c|c|c|c|}
\hline Autores & \begin{tabular}{|l|} 
Faixa Etária / Amostra \\
(Critério de Inclusão)
\end{tabular} & $\begin{array}{c}\begin{array}{c}\text { Período/Desenho } \\
\text { do Estudo }\end{array} \\
\end{array}$ & Mortalidade & $\begin{array}{l}\text { Análise Estatística e Fatores Relacionados com Pior } \\
\text { Prognóstico }\end{array}$ \\
\hline $\begin{array}{l}\text { Ducrocq e } \\
\text { col. }^{43}\end{array}$ & $\begin{array}{l}1 \text { mês a } 15 \text { anos } \\
585 \text { pacientes } \\
(E C G \leq 8)\end{array}$ & $\begin{array}{c}9 \text { anos } \\
\text { retrospectivo }\end{array}$ & $22 \%$ & $\begin{array}{l}\text { Análise multivariada: } \mathrm{ECG}^{+} \text {, ISS }{ }^{\star} \text {, tipo de lesão intra- } \\
\text { craniana, hipotensão e idade }<2 \text { anos. }\end{array}$ \\
\hline $\begin{array}{l}\text { Campbell } \\
\text { e col. }{ }^{44}\end{array}$ & $\begin{array}{l}1 \text { a } 18 \text { anos } \\
83 \text { pacientes } \\
(E C G \leq 12) \\
\end{array}$ & $\begin{array}{c}5 \text { anos } \\
\text { retrospectivo }\end{array}$ & $13 \%$ & Análise univariada: idade, $\mathrm{ECG}^{+}, \mathrm{PRISM}^{\epsilon}$ \\
\hline $\begin{array}{l}\text { Hackbarth } \\
\text { e col. }{ }^{45}\end{array}$ & $\begin{array}{l}1 \text { mês a } 18 \text { anos } \\
79 \text { pacientes } \\
(E C G \leq 10)\end{array}$ & $\begin{array}{c}4 \text { anos } \\
\text { retrospectivo }\end{array}$ & $23 \%$ & $\begin{array}{l}\text { Análise univariada: ECG, hipotensão, bradicardia, } \\
\text { ETP }{ }^{\sharp} \text {, baixa PPE\# } \\
\text { Multivariada: bradicardia na sala de emergência e bai- } \\
\text { xa PPE na UTI }\end{array}$ \\
\hline $\begin{array}{l}\text { Chiaretti e } \\
\text { col. }{ }^{25}\end{array}$ & $\begin{array}{l}\text { Idade média } 122,7 \pm 45,9 \text { meses } \\
122 \text { pacientes } \\
(E C G \leq 8)\end{array}$ & $\begin{array}{c}8 \text { anos } \\
\text { retrospectivo }\end{array}$ & $14,7 \%$ & $\begin{array}{l}\text { Análise univariada e multivariada: ECG, politrauma, } \\
\text { crise convulsiva, hiperglicemia, hipóxia/hipotensão, } \\
\text { CIVD\#\#. }\end{array}$ \\
\hline $\begin{array}{l}\text { Vavilala e } \\
\text { col. }{ }^{26}\end{array}$ & $\begin{array}{l}0 \text { a } 14 \text { anos } \\
172 \text { pacientes } \\
\text { (ECG } \leq 8 \text { e lesão cerebral difusa) }\end{array}$ & $\begin{array}{l}5 \text { anos } \\
\text { retrospectivo }\end{array}$ & $17 \%$ & $\begin{array}{l}\text { Análise univariada e multivariada: hipotensão, hipoter- } \\
\text { mia e agressão. }\end{array}$ \\
\hline $\begin{array}{l}\text { Pfen- } \\
\text { ninger e } \\
\text { col. }{ }^{27}\end{array}$ & $\begin{array}{l}1 \text { mês a } 16 \text { anos } \\
51 \text { pacientes } \\
(E C G \leq 8)\end{array}$ & $\begin{array}{l}5 \text { anos } \\
\text { retrospectivo }\end{array}$ & $27,5 \%$ & $\begin{array}{l}\text { Análise univariada: ECG, pupilas fixas e dilatadas, cis- } \\
\text { ternas de base comprimidas, coagulopatia, } \mathrm{HIC}^{\star *} \text {. }\end{array}$ \\
\hline $\begin{array}{l}\text { Maldaun e } \\
\text { col. }{ }^{46}\end{array}$ & $\begin{array}{l}3 \text { meses a } 14 \text { anos } \\
52 \text { pacientes } \\
\text { (internação em UTI) }\end{array}$ & $\begin{array}{l}2 \text { anos } \\
\text { retrospectivo }\end{array}$ & $11,5 \%$ & $\begin{array}{l}\text { Análise univariada: ECG, hipotensão arterial, altera- } \\
\text { ções tomográficas, HIC }\end{array}$ \\
\hline $\begin{array}{l}\text { White e } \\
\text { col. }^{5}\end{array}$ & $\begin{array}{l}0 \text { a } 17 \text { anos } \\
136 \text { pacientes } \\
(E C G \leq 8)\end{array}$ & $\begin{array}{l}5 \text { anos } \\
\text { retrospectivo }\end{array}$ & $24 \%$ & $\begin{array}{l}\text { Análise univariada: ISS*, ECG, PA sistólica, HIC, tem- } \\
\text { peratura corporal baixa, } \mathrm{PaCO}_{2} \text { baixo, TC alterada, he- } \\
\text { morragia subaracnóidea ou subdural, edema cerebral, } \\
\text { LAD. Multivariada: ECG, PA sistólica. }\end{array}$ \\
\hline $\begin{array}{l}\text { Pillai e } \\
\text { col. }{ }^{47}\end{array}$ & $\begin{array}{l}0 \text { a } 15 \text { anos } \\
74 \text { pacientes } \\
\text { (ECG } \leq 8 \text { e lesão cerebral difusa) }\end{array}$ & $\begin{array}{c}7 \text { anos } \\
\text { retrospectivo }\end{array}$ & $56,8 \%$ & $\begin{array}{l}\text { Análise univariada: ECG, ausência de reação pupilar, } \\
\text { ausência de reflexo óculo-cefálico, hemorragia suba- } \\
\text { racnóidea traumática. } \\
\text { Multivariada: reflexo óculo-cefálico, ECG. }\end{array}$ \\
\hline $\begin{array}{l}\text { Downard } \\
\text { e col. }{ }^{36}\end{array}$ & $\begin{array}{l}0 \text { a } 15 \text { anos } \\
118 \text { pacientes } \\
\text { (monitorização da } \mathrm{PIC}^{++} \text {) }\end{array}$ & $\begin{array}{l}8 \text { anos } \\
\text { retrospectivo }\end{array}$ & $28 \%$ & $\begin{array}{l}\text { Análise univariada: ECG, hipernatremia. } \\
\text { Multivariada: PPE menor que } 40 \mathrm{mmHg} \text {, PIC maior que } \\
20 \mathrm{mmHg}\end{array}$ \\
\hline $\begin{array}{l}\text { Feickert e } \\
\text { col. }{ }^{16}\end{array}$ & $\begin{array}{l}0 \text { a } 16 \text { anos } \\
150 \text { pacientes } \\
(E C G \leq 8)\end{array}$ & $\begin{array}{c}17 \text { anos } \\
\text { retrospectivo }\end{array}$ & $22 \%$ & $\begin{array}{l}\text { Análise univariada e multivariada: ECG, arreflexia e } \\
\text { edema cerebral. }\end{array}$ \\
\hline $\begin{array}{l}\text { Thakker e } \\
\text { col. }{ }^{48}\end{array}$ & $\begin{array}{l}1 \text { mês a } 14 \text { anos } \\
105 \text { pacientes } \\
\text { (sob intubação traqueal) }\end{array}$ & $\begin{array}{c}5 \text { anos } \\
\text { retrospectivo }\end{array}$ & $16,2 \%$ & $\begin{array}{l}\text { Análise univariada: idade, ECG, PRISM, taquicardia e } \\
\text { hipotensão. } \\
\text { Multivariada: idade, PRISM, taquicardia e hipotensão. }\end{array}$ \\
\hline
\end{tabular}


Continuação Tabela 1 - Estudos sobre os Fatores Relacionados com Mortalidade de Crianças e Adolescentes com Trauma Cranioencefálico Grave (1985-2006).

\begin{tabular}{|c|c|c|c|c|}
\hline $\begin{array}{l}\text { Song e } \\
\text { col. }{ }^{15}\end{array}$ & $\begin{array}{l}5 \text { meses a } 15 \text { anos } \\
73 \text { pacientes } \\
(E C G \leq 8)\end{array}$ & $\begin{array}{c}5 \text { anos } \\
\text { retrospectivo }\end{array}$ & $23 \%$ & $\begin{array}{l}\text { Análise univariada: ECG, postura motora anormal, altera- } \\
\text { ções pupilares, hematoma subdural agudo. }\end{array}$ \\
\hline $\begin{array}{l}\text { Pigula e } \\
\text { col. }^{34}\end{array}$ & $\begin{array}{l}0 \text { a } 16 \text { anos } \\
58 \text { pacientes } \\
(E C G \leq 8)\end{array}$ & $\begin{array}{c}5 \text { anos } \\
\text { prospectivo }\end{array}$ & $29 \%$ & $\begin{array}{l}\text { Análise univariada e multivariada: hipotensão com ou } \\
\text { sem hipóxia, ECG, ISS. }\end{array}$ \\
\hline $\begin{array}{l}\text { Michaud e } \\
\text { col. }^{14}\end{array}$ & $\begin{array}{l}0 \text { a } 16 \text { anos } \\
75 \text { pacientes } \\
(E C G \leq 8)\end{array}$ & $\begin{array}{l}2 \text { anos } \\
\text { transversal }\end{array}$ & $33 \%$ & $\begin{array}{l}\text { Análise univariada: resposta pupilar, hipotensão, bra- } \\
\text { dicardia, ISS, HIC } \\
\text { Multivariada: ISS, resposta pupilar }\end{array}$ \\
\hline $\begin{array}{l}\text { Elias- } \\
\text { Jones e } \\
\text { col. }^{24} \\
\end{array}$ & $\begin{array}{l}2 \text { meses a } 13 \text { anos } \\
39 \text { pacientes } \\
(?)\end{array}$ & $\begin{array}{l}5 \text { anos } \\
(?)\end{array}$ & $23 \%$ & Análise univariada: PPE, HIC, hipocapnia. \\
\hline $\begin{array}{l}\text { Lieh-Lai e } \\
\text { col. }^{49}\end{array}$ & $\begin{array}{l}1 \text { mês a } 17 \text { anos } \\
79 \text { pacientes } \\
\text { (admissão em UTI) }\end{array}$ & $\begin{array}{l}4 \text { anos } \\
\text { retrospectivo }\end{array}$ & $11 \%$ & $\begin{array}{l}\text { Análise univariada: ECG, } \mathrm{PCR}^{\&}, \mathrm{HIC} \text {, achados tomo- } \\
\text { gráficos (hemorragia intraventricular ou subaracnóidea } \\
\text { ou desvio de linha média) }\end{array}$ \\
\hline $\begin{array}{l}\text { Alberico e } \\
\text { col. }^{12}\end{array}$ & $\begin{array}{l}0 \text { a } 19 \text { anos } \\
100 \text { pacientes } \\
(E C G \leq 8) \\
\end{array}$ & $\begin{array}{c}8 \text { anos } \\
\text { prospectivo }\end{array}$ & $24 \%$ & Análise univariada: idade e lesões focais à tomografia \\
\hline $\begin{array}{l}\text { Mayer e } \\
\text { col. }^{20}\end{array}$ & $\begin{array}{l}3 \text { semanas a } 16 \text { anos } \\
200 \text { pacientes } \\
(E C G \leq 8)\end{array}$ & $\begin{array}{c}4 \text { anos } \\
\text { prospectivo }\end{array}$ & $21,5 \%$ & $\begin{array}{l}\text { Análise univariada: ECG, HIC, hipotensão, hipóxia ou } \\
\text { hipercarbia, múltiplos traumas. }\end{array}$ \\
\hline $\begin{array}{l}\text { Berger e } \\
\text { col. }{ }^{21}\end{array}$ & $\begin{array}{l}0 \text { a } 18 \text { anos } \\
37 \text { pacientes } \\
(E C G \leq 8)\end{array}$ & $\begin{array}{l}5 \text { anos } \\
\text { prospectivo }\end{array}$ & $33 \%$ & Análise univariada: lesões focais à tomografia, HIC \\
\hline
\end{tabular}

+ECG - Escala de Coma de Glasgow

EPRISM - Pediatric Risk of Mortality

${ }^{*}$ ETP - Escala de Trauma Pediátrico

\#PPE - Pressão de Perfusão Encefálica

**HIC - Hipertensão Intracraniana

\#CIVD - Coagulação Intravascular Disseminada

*ISS - Injury Severity Score

${ }^{++}$PIC - Pressão Intracraniana

\&PCR - Parada cardiorrespiratória

\section{TRATAMENTO}

A avaliação e a abordagem inicial da criança na sala de emergência são constituídas por procedimentos simples, que, se feitos de forma rápida e ordenada, trarão grande benefício ao paciente. A seqüência de atendimento prioriza as lesões que levariam ao óbito mais rapidamente e, por isso, devem ser tratadas em primeiro lugar 6 ,

$O$ atendimento inicial compõe-se de:

A - Abordagem das vias aéreas com imobilização da coluna cervical;

B - Ventilação adequada, visando à normocapnia;

C - Abordagem da circulação e controle de sangramentos externos;

$D$ - Exame neurológico: ECG, pupilas, movimento dos quatro membros;

E - Exposição e avaliação de todo o corpo.

Até o ano de 2003, as condutas para tratamento do TCE grave em crianças e adolescentes eram baseadas nas diretrizes da Brain Trauma Foundation para adultos $^{30}$. Naquele ano, foram publicadas as primeiras diretrizes para o tratamento das crianças e dos adolescentes ${ }^{31}$ :

- Triagem: em regiões metropolitanas, as crianças com TCE grave devem ser transportadas diretamente para um centro de trauma pediátrico, podendo, opcionalmente, serem atendidas em um centro de trauma de adultos com qualificação para atendimento pediátrico ${ }^{32}$;

- Atendimento inicial: (Fluxograma 1) em toda criança com pontuação na ECG menor ou igual a oito deve-se assegurar via aérea definitiva, a fim de se evitar a hipóxia, a hipercarbia e a aspiração $0^{14,20}$. A freqüência de complicações nos procedimentos para obtenção da via aérea justifica a utilização de protocolos com medicações para proteção encefálica, anestesia, analgesia e bloqueio neuromuscular. A seqüência rápida de intubação é recomendada para evitar aumento da pressão intracraniana (PIC) que ocorre durante a laringoscopia e pela passagem do tubo pela glote. A lidocaína também pode ser utilizada com o mesmo intuito. Após a intubação deve-se manter a $\mathrm{PaCO}_{2}$ de 35 a $40 \mathrm{mmHg}$. O uso 
A. Corrigir hipoxemia $\left(\mathrm{SatO}_{2} \geq 95 \%\right)$

B. Normocapnia $\left(\mathrm{PaCO}_{2} \pm 35 \mathrm{mmHg}\right)$

C. $\mathrm{PAS} \geq 90 \mathrm{mmHg}+(2 \times$ idade $) /$ percentil 50

D. ECG + pupilas + movimentação 4 membros

E. Outras lesões graves / sangramentos? / manter normotermia

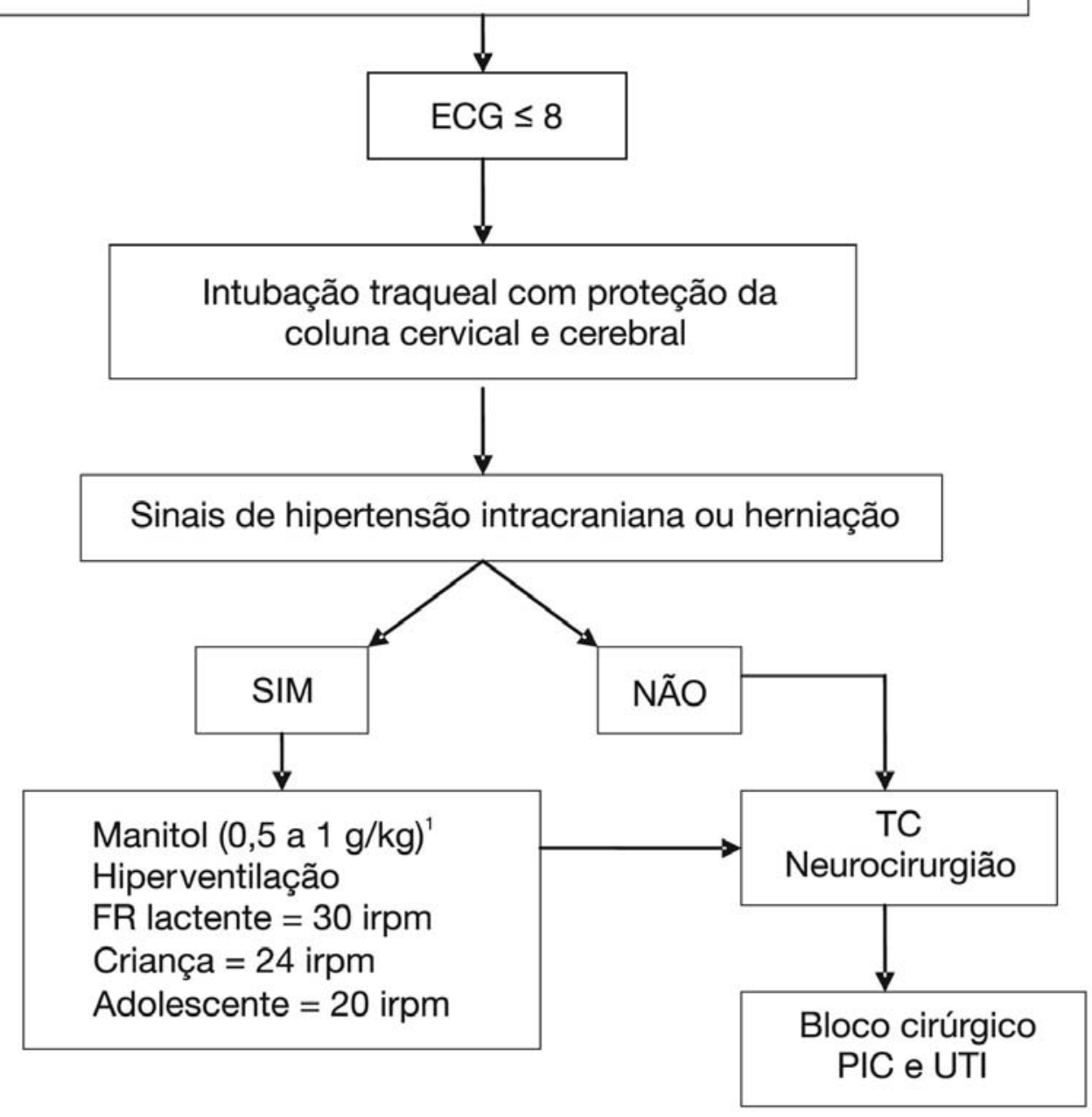

Sinais de Herniação ou HIC

- Pupilas (dilatação pupilar unilateral)

- Resposta motora (plegia, descerebração ou decorticação unilaterais)

- Tríade de Cushing (hipertensão arterial, bradicardia e alterações respiratórias)

- Queda em 3 ou mais pontos na ECG

- PCR súbita

1Opção: pode-se usar solução salina a 3\% para pacientes hemodinamicamente instáveis

ECG - Escala de coma de Glasgow FR - Freqüência respiratória

HIC - Hipertensão Intracraniana PAS - Pressão arterial sistólica

PCR - Parada cardiorrespiratória PIC - Pressão Intracraniana

TC - Tomografia Computadorizada 
de sedativos, analgésicos e bloqueadores neuromusculares podem ser úteis durante o transporte ${ }^{33}$;

- A hipotensão deve ser identificada e corrigida imediatamente, por meio de reposição volêmica ${ }^{14,23,34}$. Ainda existem divergências nos estudos até então publicados quanto aos níveis pressóricos mais apropriados na criança com TCE grave , $^{5,27}$;

As medidas específicas para redução da pressão intracraniana somente devem ser realizadas no atendimento inicial se o paciente apresentar sinais de hipertensão intracraniana (HIC), de herniação transtentorial ou piora neurológica ${ }^{33}$. Estes sinais são: tríade de Cushing (hipertensão arterial, bradicardia e alterações respiratórias), dilatação pupilar unilateral ou pupilas fixas e dilatadas bilateralmente, plegia ou postura motora de decorticação ou descerebração, principalmente unilaterais, diminuição de três ou mais pontos na ECG ou ocorrência de parada cardiorrespiratória súbita. As medidas a serem tomadas são: administração de manitol - 0,5 a 1 g/kg, em bolus, intubação e hiperventilação moderada (manter $\mathrm{PaCO}_{2} \pm 30 \mathrm{mmHg}$ ) ${ }^{31}$. Para aqueles pacientes que estejam hemodinamicamente instáveis, pode-se considerar o uso de solução salina hipertônica a $3 \%$ em infusão contínua a 0,1 a $1 \mathrm{~mL} / \mathrm{kg} /$ hora $^{35}$; Após a estabilização inicial, deve-se avaliar e anotar o mecanismo de trauma; ocorrência de crise convulsiva; perda de consciência (tempo); ocorrência de cefaléia, tonteira, náuseas ou vômitos. No exame secundário, deve ser observado a existência de hematomas no couro cabeludo, lesões contusas de crânio ou face, edemas e sinais de fratura de base de crânio (equimose periorbitária ou retroauricular, escape de líquor ou sangue pelo nariz ou pelo ouvido ou coleção de sangue retro-timpânica) ${ }^{8}$.

A tomografia computadorizada de crânio e encéfalo deverá ser realizada o mais rapidamente possível. Devem-se realizar exames laboratoriais como: hemograma, glicemia, ionograma, gasometria arterial, atividade de protrombina, tempo de protrombina, tempo parcial de tromboplastina ativada, número de plaquetas e dosagem de fibrinogênio.

- Monitorização da pressão intracraniana (PIC): a hipertensão intracraniana é de difícil diagnóstico em lactentes e crianças pequenas e está associada com aumento da mortalidade e pior recuperação neurológi$\mathrm{ca}^{11,20,21,24,27,36}$. É apropriada a monitorização da PIC em toda criança com TCE grave com ECG $\leq 8^{31}$. A ausência de alterações tomográficas em pacientes comatosos não exclui a possibilidade de hipertensão intracraniana $^{37}$, principalmente em pacientes com hipotensão arterial ou posturas motora anormal, unilateral ou bilatera $\left.\right|^{38}$. A presença de suturas ou fontanelas abertas nos lactentes não impede a ocorrência de hipertensão intracraniana e, dessa forma, não dispensa a monitorização da PIC ${ }^{39}$. A monitorização deve ser avaliada nas crianças com TCE leve ou moderado que apresentem lesões intracranianas com efeito de massa ou naquelas em que o exame neurológico seriado esteja prejudicado em razão da sedação, do bloqueio neuromuscular ou da anestesia ${ }^{31}$;

- Tratamento da hipertensão intracraniana (HIC): deve ser iniciado quando a PIC for maior ou igual a $20 \mathrm{mmHg}$ (Fluxograma 2). O tratamento e a interpretação da PIC devem ser baseados no exame clínico seriado, na monitorização dos parâmetros fisiológicos e nas imagens tomográficas ${ }^{31}$;

- Pressão de perfusão encefálica (PPE): é a diferença entre a pressão arterial média (PAM) e a PIC e deve ser mantida em valores acima de $40 \mathrm{mmHg}$ em crianças com TCE grave. Provavelmente uma PPE entre 40 e $65 \mathrm{mmHg}$, dependendo da idade, pode representar o melhor tratamento 24,36 ;

- Uso de sedativos e bloqueadores neuromusculares: não há estudos que comprovem que seu uso seja eficaz no tratamento de crianças com TCE grave. O seu efeito no controle da HIC é variável ${ }^{31}$;

- Drenagem liqüórica: deve ser considerada como uma opção para o tratamento da $\mathrm{HIC}^{31}$;

- Soluções hiperosmolares: solução salina hipertônica a $3 \%$ em doses de 0,1 a $1 \mathrm{~mL} / \mathrm{kg} /$ hora ou manitol em doses de 0,25 a $1 \mathrm{~g} / \mathrm{kg}$ podem ser utilizados para controle da HIC. A osmolaridade sérica deve estar menor que $320 \mathrm{mOsm} / \mathrm{L}$ para utilização do manitol e menor que 360 mOsm/L para utilização da solução salina hipertônica ${ }^{31,35}$;

- Hiperventilação: a hiperventilação profilática $\left(\mathrm{PaCO}_{2}\right.$ menor que $35 \mathrm{mmHg}$ ) deve ser evitada. A hiperventilação moderada $\left(\mathrm{PaCO}_{2}\right.$ de 30 a $\left.35 \mathrm{mmHg}\right)$ pode ser utilizada para controle da HIC que não respondeu ao uso de sedação, analgesia, bloqueio neuromuscular, drenagem liqüórica e terapia hiperosmolar. A hiperventilação agressiva $\left(\mathrm{PaCO}_{2}\right.$ menor que $\left.30 \mathrm{mmHg}\right)$ somente deve ser utilizada, como tratamento de segunda linha, nos casos de HIC refratária ou por curtos períodos de tempo, nos casos de herniação cerebral ou piora neurológica aguda ${ }^{30,31}$;

- Coma barbitúrico: sua utilização pode ser considerada nos pacientes hemodinamicamente estáveis com HIC refratária. O fármaco utilizado é o tiopental, em dose de ataque $(10 \mathrm{mg} / \mathrm{kg})$ e em dose de manutenção 


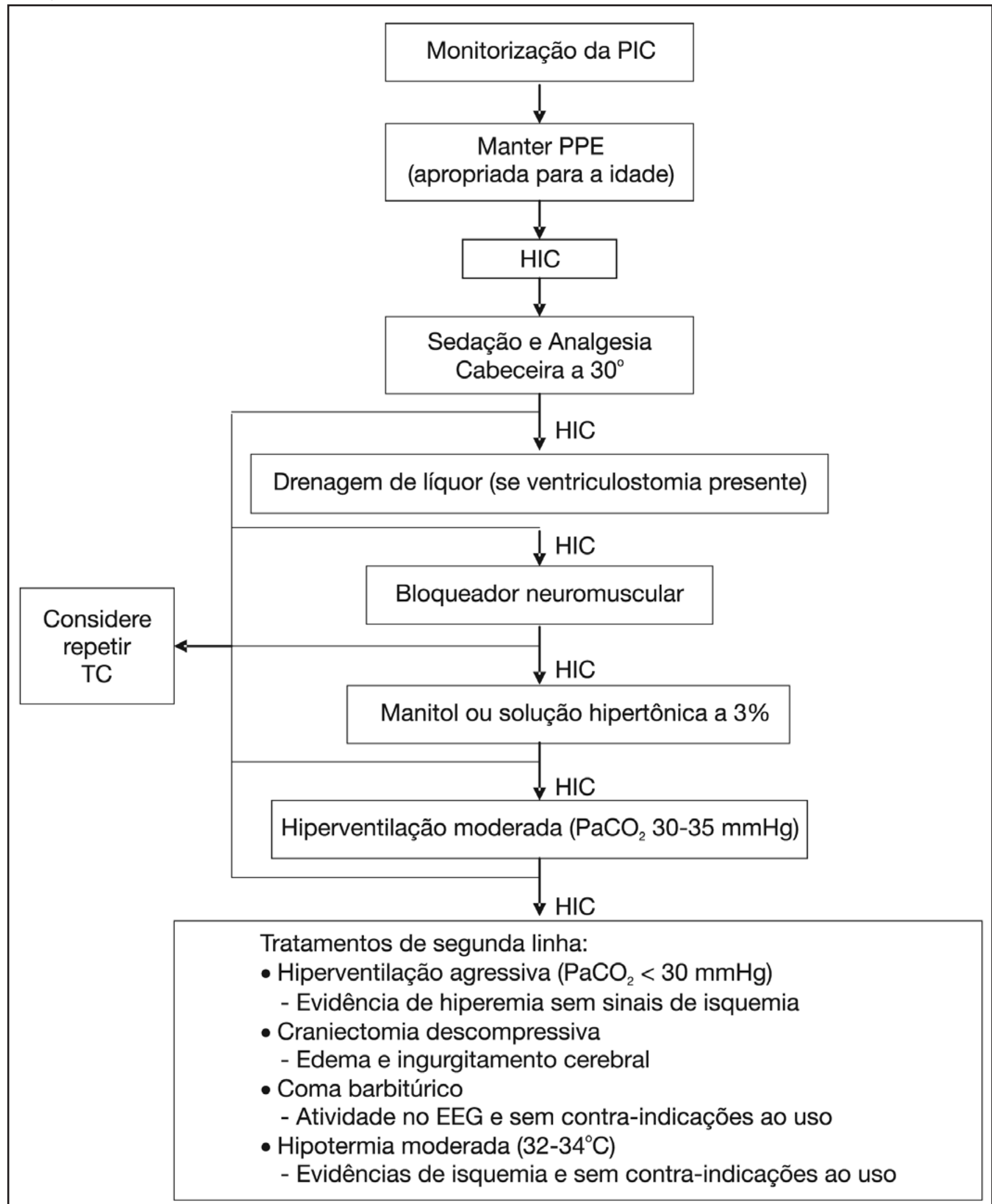


(1 a $5 \mathrm{mg} / \mathrm{kg} / \mathrm{hora})^{40}$;

- Temperatura corporal: a hipertermia deve ser evitada e corrigida. Apesar dos poucos estudos em crianças, a hipotermia controlada pode ser utilizada para o tratamento da HIC refratária ${ }^{41}$;

- Craniectomia descompressiva: pode ser realizada em crianças com TCE grave e HIC refratária, que preencham alguns ou todos os critérios a seguir ${ }^{31}$ :

1. Tomografia com edema e ingurgitamento cerebral difusos;

2. Primeiras 48 horas de trauma;

3. Ausência de episódios de PIC maior que $40 \mathrm{mmHg}$, por período prolongado;

4. Pontuação maior que três na ECG, em alguma avaliação durante a internação;

5. Piora clínica secundária;

6. Síndrome de herniação cerebral.

- Corticoterapia: não está indicada, em virtude da falta de evidências de efeito benéfico e do risco de potenciais complicações ${ }^{31}$;

- Profilaxia de crises convulsivas: está indicado o uso de fenitoína durante sete dias ${ }^{31,42}$.

\section{CONCLUSÃO}

Apesar do grande número de trabalhos publicados, os fatores relacionados com um melhor prognóstico em crianças ainda permanecem obscuros, havendo algumas divergências entre os estudos. Grande parte destes fatores é corrigível ou evitável, com reanimação hídrica inicial agressiva, tratamento cirúrgico em tempo hábil, monitoração e cuidados intensivos adequados.

\section{REFERÊNCIAS}

01. Carli P, Orliaguet G - Severe traumatic brain injury in children. Lancet, 2004;363:584-585.

02. Ministério da Saúde, 2000. Acessado em 01 de julho de 2003. Disponível em: http://www.datasus.gov.br.

03. Cantor RM, Leaming JM - Evaluation and management of pediatric major trauma. Emerg Med Clin North Am, 1998;16:229-256.

04. Allen EM, Boyer R, Cherny WB et al - Head and Spinal Cord Injury, em: Rogers MC - Textbook of Pediatric Intensive Care. $3^{\text {rd }}$ Ed, Baltimore, Williams \& Wilkins, 1996;809-857.

05. White JR, Farukhi Z, Bull C et al - Predictors of outcome in severely head-injured children. Crit Care Med, 2001;29:534-540.

06. Guerra SD, Jannuzzi MA, Moura AD - Traumatismo cranioencefálico em pediatria. J Pediatr, 1999;75:(Suppl2):S279-S293.

07. Marik PE, Varon J, Trask T - Management of head trauma. Chest, 2002;122:699-711.

08. American College of Surgeons Committee on Trauma. ATLS Course manual. Chicago, IL: American College of Surgeons, 1997.

09. Mazzola CA, Adelson PD - Critical care management of head trauma in children. Crit Care Med, 2002;30(Suppl11):S393-S401.

10. Maas Al, Dearden M, Teasdale GM et al - EBIC - EBIC-guidelines for management of severe head injury in adults. Acta Neurochir (Wien),
1997;139:286-294.

11. Chesnut RM, Marshall LF, Klauber MR et al - The role of secondary brain injury in determining outcome from severe head injury. J Trauma, 1993;34:216-222.

12. Alberico AM, Ward JD, Choi SC et al - Outcome after severe head injury. Relationship to mass lesions, diffuse injury, and ICP course in pediatric and adult patients. J Neurosurg, 1987;67:648-656.

13. Luerssen TG, Klauber MR, Marshall LF - Outcome from head injury related to patient's age. A longitudinal prospective study of adult and pediatric head injury. J Neurosurg, 1988;68:409-416.

14. Michaud LJ, Rivara FP, Grady MS et al - Predictors of survival and severity of disability after severe brain injury in children. Neurosurgery, 1992;31:254-264.

15. Song $\mathrm{SH}, \mathrm{Kim} \mathrm{SH}$, Kim KT et al - Outcome of pediatric patient with severe brain injury in Korea: a comparison with reports in the west. Childs Nerv Syst, 1997;13:82-86.

16. Feickert H, Drommer S, Heyer R - Severe head injury in children: impact of risk factors on outcome. J Trauma, 1999;47:33-38.

17. Bruce DA, Raphaely RC, Goldberg Al et al - Pathophysiology, treatment and outcome following severe head injury in children. Childs Brain, 1979;5:174-191.

18. Alberico AM, Ward JD, Choi SC et al - Outcome after severe head injury. Relationship to mass lesions, diffuse injury, and ICP course in pediatric and adult patients. J Neurosurg, 1987;67:648-656.

19. Bruce DA, Schut L, Bruno LA et al - Outcome following severe head injuries in children. J Neurosurg, 1978;48:679-688.

20. Mayer TA, Walker ML - Pediatric head injury: the critical role of the emergency physician. Ann Emerg Med, 1985;14:1178-1184.

21. Berger MS, Pitts LH, Lovely $M$ et al - Outcome from severe head injury in children and adolescents. J Neurosurg, 1985;62:194-199.

22. Teasdale G, Jennett B - Assessment of coma and impaired consciousness. A pratical scale. Lancet, 1974;2:81-84.

23. Kokoska ER, Smith GS, Pittman T et al - Early hypotension worsens neurological outcome in pediatric patients with moderately severe head trauma. J Pediatr Surg, 1998;33:333-338.

24. Elias-Jones AC, Punt JA, Turnbull AE et al - Management and outcome of severe head injuries in the Trent region, 1985-1990. Arch Dis Child, 1992:67:1430-1435.

25. Chiaretti A, Piastra M, Pulitano S et al - Prognostic factors and outcome of children with severe head injury: an 8-year experience. Childs Nerv Syst, 2002;18:129-136.

26. Vavilala MS, Bowen A, Lam AM et al - Blood pressure and outcome after severe pediatric traumatic brain injury. J Trauma, 2003;55:1039-1044.

27. Pfenninger J, Santi A - Severe traumatic brain injury in children - are the results improving? Swiss Med Wkly, 2002;132:116-120.

28. Eisenberg HM, Gary HE Jr, Aldrich EF et al - Initial CT findings in 753 patients with severe head injury. A report from the NIH Traumatic Coma Data Bank. J Neurosurg, 1990;73:688-698.

29. Ong L, Selladurai BM, Dhillon MK et al - The prognostic value of the Glasgow Coma Scale, hypoxia and computerised tomography in outcome prediction of pediatric head injury. Pediatr Neurosurg, 1996;24:285291.

30. Bullock MR, Chesnut RM, Clifton GL et al - Guidelines for the management of severe traumatic brain injury. J Neurotrauma, 2000;17:451-553.

31. Adelson PD, Bratton SL, Carney NA et al - Guidelines for the acute medical management of severe traumatic brain injury in infants, children, and adolescents. Pediatr Crit Care Med, 2003;4:(Suppl3):S1-S75.

32. Potoka DA, Schall LC, Gardner MJ et al - Impact of pediatric trauma centers on mortality in a statewide system. J Trauma, 2000;49:237-245.

33. Gabriel ES, Ghajar J, Jagoda A et al - Guidelines for prehospital management of traumatic brain injury. J Neurotrauma, 2002;19:111-174.

34. Pigula FA, Wald SL, Shackford SR et al - The effect of hypotension and hypoxia on children with severe head injuries. J Pediatr Surg, 1993;28:310-316.

35. Peterson B, Khanna S, Fisher B et al - Prolonged hypernatremia controls elevated intracranial pressure in head-injured pediatric patients. Crit Care Med, 2000;28:1136-1143.

36. Downard C, Hulka F, Mullins RJ et al - Relationship of cerebral perfusion pressure and survival in pediatric brain-injured patients. J Trauma, 2000;49:654-659.

37. O'Sullivan MG, Statham PF, Jones PA et al - Role of intracranial pressure monitoring in severely head-injured patients without signs of intra- 
cranial hypertension on initial computerized tomography. J Neurosurg, 1994;80:46-50.

38. Narayan RK, Kishore PR, Becker DP et al - Intracranial pressure: to monitor or not to monitor? A review of our experience with severe head injury. J Neurosurg, 1982;56:650-659.

39. Cho DY, Wang YC, Chi CS - Decompressive craniotomy for acute shaken/impact baby syndrome. Pediatr Neurosurg, 1995;23:192-198.

40. Eisenberg HM, Frankowski RF, Contant CF et al - High-dose barbiturate control of elevated intracranial pressure in patients with severe head injury. J Neurosurg, 1988;69:15-23.

41. Shafi NI, Mariscalco MM - Considering the use of induced hypothermia in a pediatric patient with traumatic brain injury: a critical appraisal of two meta-analyses. Pediatr Crit Care Med, 2006;7:468-472.

42. Young B, Rapp RP, Nprtpn JA et al - Failure of prophylactically administered phenytoin to prevent post-traumatic seizures in children. Childs Brain, 1983;10:185-192.

43. Ducrocq SC, Meyer PG, Orliaguet GA et al - Epidemiology and early predictive factors of mortality and outcome in children with traumatic severe brain injury: experience of a French pediatric trauma center. Pediatr Crit Care Med, 2006;7:461-467.
44. Campbell CG, Kuehn SM, Richards PM et al - Medical and cognitive outcome in children with traumatic brain injury. Can J Neurol Sci, 2004;31:213-219.

45. Hackbarth RM, Rzeszutko KM, Sturm G et al - Survival and functional outcome in pediatric traumatic brain injury: a retrospective review and analysis of predictive factors. Crit Care Med, 2002;30:1630-1635.

46. Maldaun MVC, Zambelli HJL, Dantas VP et al - Análise de 52 pacientes com traumatismo de crânio atendidos em UTI pediátrica: considerações sobre o uso de monitorização da pressão intracraniana. Arq Neuropsiquiatr, 2002;60:967-970.

47. Pillai S, Praharaj SS, Mohanty A et al - Prognostic factors in children with severe diffuse brain injuries: a study of 74 patients. Pediatr Neurosurg, 2001;34:98-103.

48. Thakker JC, Splaingard M, Zhu J et al - Survival and functional outcome of children requiring endotracheal intubation during therapy for severe traumatic brain injury. Crit Care Med, 1997;25:1396-1401.

49. Lieh-Lai MW, Theodorou AA, Sarnaik AP et al - Limitations of the Glasgow Coma Scale in predicting outcome in children with traumatic brain injury. J Pediatr, 1992;120:195-199. 